\title{
IMPACTO DO DESENVOLVIMENTO ECONÔMICO NAS EMISSÕES DE CO2: UMA APLICAÇÃO DA CURVA AMBIENTAL DE KUZNETS PARA O MATO GROSSO DO SUL
}

\author{
Simone Yukimi Kunimoto \\ Mestre em Desenvolvimento Local pela Universidade Católica Dom Bosco (2018). Pós- \\ Graduada em Gestão Empresarial e Recursos Humanos pelo Centro Universitário UNAES \\ (2008). Graduada em Administração pelo Instituto Euro-Americano de Educação, Ciência e \\ Tecnologia de Brasília (2006). \\ Email: michelangelo111@gmail.com
}

Daniel Silva Boson

\begin{abstract}
Doutor em Direito pelo UniCEUB, com distinção. Mestre em Direito pela Universidade Católica de Brasília. Especialista em Administração de Empresas e em Defesa da Concorrência, ambas pela FGV. Gestor Público Federal (EPPGG) prestando assessoria jurídico-econômica no Conselho Administrativo de Defesa Econômica (CADE). Professor de Economia e de Direito Econômico no UniCEUB. Email: daniel.boson@cade.gov.br
\end{abstract}

Michel Angelo Constantino de Oliveira

Doutor em Economia pela Universidade Católica de Brasília (UCB), Mestre em Desenvolvimento Local (UCDB) e Administrador. Professor nos Programas de Doutorado em Ciências Ambientais e Sustentabilidade Agropecuária e em Desenvolvimento Local. Pesquisador da área de Políticas Públicas Agroambientais, Economia Comportamental, Economia Regional e Econometria (Métodos Quantitativos). Email: michelangelo111@gmail.com

Dany Rafael Fonseca Mendes

Mestrado em Análise Econômica do Direito pela UCB (2014), bacharelado em Direito pela UFOP (2008). Consultor (OAB/DF 36620). Professor Adjunto do UniCEUB. Email: rafael.dany@gmail.com

\section{RESUMO}

As atividades produtivas são recorrentes causadoras de externalidades, e a literatura é controversa quanto ao real impacto que tais atividades geram ao meio ambiente, ora informando sobre externalidades negativas, ora noticiando acerca de externalidades positivas. O presente estudo analisa o impacto do desenvolvimento econômico nas emissões de dióxido de carbono $\left(\mathrm{CO}_{2}\right)$ no Mato Grosso do Sul (MS), compreendendo o período entre 2002 e 2016. Para a realização das análises, cinco setores foram investigados: agropecuária, energia, mudança e uso da terra, processos industriais e resíduos, todos relacionados com PIB (Produto Interno Bruto) per Capita de formato linear e quadrático, conforme teoria de Kuznets, como fontes de desenvolvimento econômico. A pesquisa foi exploratória, inédita e baseada em modelos econométricos. Os resultados empíricos indicam que, ao longo do período avaliado, os impactos do desenvolvimento econômico foram negativos nas emissões de dióxido de carbono totais para o Mato Grosso do Sul. O modelo econométrico de resíduos apresentou a melhor significância, sendo controverso quanto aos resultados em países desenvolvidos; para os demais modelos, os setores apresentaram o formato de "U"; e o setor de mudança e uso da terra apresentou a teoria da curva ambiental de Kuznets ("U" invertido). Quando 
comparado aos estados brasileiros industrializados, o Mato Grosso do Sul se mostra incipiente em seu desempenho econômico; porém, ao analisar a trajetória da sustentabilidade ambiental, medida pelas emissões de $\mathrm{CO}_{2}$, o MS apresenta melhores números empiricamente constatados.

Palavras-chave: Desenvolvimento Econômico; Meio Ambiente; Economia; Econometria; Modelagem Econométrica.

\title{
ECONOMIC DEVELOPMENT'S IMPACT ON CO2 EMISSION: AN APPLICATION OF ENVIRONMENTAL KUZNETS CURVE IN MATO GROSSO DO SUL
}

\begin{abstract}
Productive activities are recurrent causes of externalities, and the literature is controversial as to the real impact that these activities generate on the environment, sometimes informing about negative externalities, or reporting on positive externalities. The present study analyzes the impact of economic development on carbon dioxide (CO 2 ) emissions in Mato Grosso do Sul (MS), covering the period between 2002 and 2016.In order to carry out the analyzes, five sectors were investigated: agriculture, energy, land use change and use, industrial processes and residues, all related to GDP per capita of linear and quadratic format, according to Kuznets theory, as sources of economic development. The research was exploratory, unpublished and based on econometric models .The empirical results indicate that, during the period evaluated, the impacts of economic development were negative in the total carbon dioxide emissions for Mato Grosso do Sul. The econometric model of residues presented the best significance, being controversial about the results in countries developed; for the other models, the sectors presented the " $U$ " format; and the land use and change sector introduced the Kuznets' (" $U$ " inverted) environmental curve theory. When compared to the industrialized Brazilian states, Mato Grosso do Sul is incipient in its economic performance; However, when analyzing the environmental sustainability trajectory, as measured by CO 2 emissions, the MS presents better empirically verified numbers.
\end{abstract}

KEYWORDS: Economic Development; Environment; Economy; Econometrics; Econometric Modeling. 


\section{INTRODUÇÃO}

A sustentabilidade ambiental e o crescimento econômico são extensas fontes de pesquisa e aprofundamento de debates no âmbito da sociedade, da academia e da elaboração de políticas públicas.

Os problemas ambientais são inúmeros, e a degradação pode ser causada pela majoração de fatores como industrialização, transporte, população, pobreza, erosão do solo, congestionamento/tráfego, exploração de recursos de acesso aberto cujo direito à propriedade estejam mal definidos, e poluentes/resíduos que afetam o crescimento econômico (BORHAN et al, 2012).Os efeitos do aumento populacional são visíveis ao longo do tempo, promovendo maior poluição ambiental e contribuindo para externalidades negativas relativas ao bem-estar humano, seja no aumento dos custos sociais e de saúde, seja na produtividade.

O Protocolo de Kyoto de 1997 foi adotado na terceira Conferência das Partes $^{1}$ (COP 3) e é reconhecidamente um marco governamental comunitário voltado para a redução das emissões dos Gases de Efeito Estufa (GEE), dentre os quais o dióxido de carbono é o maior representante. Entre as metas daquele protocolo estavam a redução em 5\% da emissão dos GEE, comparados, para os países desenvolvidos, aos parâmetros de 1990. A mais recente evolução daquela primeira tentativa de acordo se concretizou na COP21, realizada na França, em 2015, com a elaboração do Acordo de Paris, por meio do qual 195 países concordaram em parametrizar suas metas voluntárias de redução de emissão de $\mathrm{CO}_{2}$, restringindo em menos de $1,5^{\circ} \mathrm{C}$ o aumento da temperatura do planeta até 2100 (MCTIC, ONU MEIO AMBIENTE, 2017).

A partir dos anos 1970, as emissões per capita de dióxido de carbono no Brasil avançaram, em média, 1,6\% ao ano. Nos anos 1990, a taxa de emissão incluiu o indicador denominado Mudança do Uso da Terra e da Floresta, que capacita a estratificação dos setores responsáveis pelas emissões de $\mathrm{CO}_{2}$. Segundo o Sistema de Estimativas de Emissões de Gases de Efeito Estufa², a partir deste indicador, é possível observar as

\footnotetext{
1 “A Conferência das Partes (COP) é o órgão supremo da Convenção Quadro das Nações Unidas sobre Mudança do Clima (UNFCCC), que reúne anualmente os países Parte em conferências mundiais. Suas decisões, coletivas e consensuais, só podem ser tomadas se forem aceitas unanimemente pelas Partes, sendo soberanas e valendo para todos os países signatários. Seu objetivo é manter regularmente sob exame e tomar as decisões necessárias para promover a efetiva implementação da Convenção e de quaisquer instrumentos jurídicos que a COP possa adotar" (MMA, 2017).

2 O SEEG "é uma iniciativa do Observatório do Clima, que compreende a produção de estimativas
} 
implicações do agronegócio na composição das emissões de dióxido de carbono. Em 2013, o Brasil possuía uma taxa de emissão de 2,4 toneladas de $\mathrm{CO}_{2}$ por habitante e, no ano seguinte, considerando-se seus números absolutos (aproximadamente $501 \mathrm{Kt}$ ), o país alcançou o décimo lugar entre os maiores emissores de dióxido de carbono no mundo (SEEG, 2017).

$\mathrm{O}$ presente estudo investiga a relação entre desenvolvimento econômico e emissões de $\mathrm{CO}_{2}$ no estado do Mato Grosso do Sul, entre 2002 e 2016. A escolha por tal campo empírico se lastreou no fato de que a referida unidade da federação reflete uma posição importante dentro da matriz econômica brasileira - com destaque para o agronegócio - atividade de forte impacto sobre o desenvolvimento local e o meio ambiente - e, ainda, por abrigar $70 \%$ do pantanal brasileiro, área peculiar com a maior área úmida continental do planeta (UNESCO, 2000) $)^{3}$. Já o recorte histórico se justifica, por ter como marco referencial a Rio+10, ocorrida em 2002 (na qual se realizou um balanço dos avanços e das novas demandas surgidas após a Rio-92) e 2016, como ano-base mais recente disponível, com dados de caráter econômico obtidos nas bases de dados do Banco Central do Brasil (BACEN). Considerando-se o parâmetro de análise para a presente pesquisa, foi adotado o mapeamento das emissões de gás carbônico, seguindo-se o raciocínio de que, segundo o Greenpeace (2009), este balanço representaria um relevante indicador do desenvolvimento econômico.

Para examinar o impacto do desenvolvimento econômico no particular analisado, foram utilizados o Produto Interno Bruto per capita e o PIB per capita ao quadrado. Para compor os dados da série de tempo de $\mathrm{CO}_{2}$ no Mato Grosso do Sul, as emissões de dióxido de carbono foram divididas por setor, mediante a elaboração de um modelo econométrico de análise, aplicando modelagem com método linear (Ordinary Least Squares

anuais das emissões de gases de efeito estufa (GEE) no Brasil, documentos analíticos sobre a evolução das emissões e um portal na internet para disponibilização de forma simples e clara dos métodos e dados gerados no sistema. As Estimativas de Emissões de Gases do Efeito Estufa são geradas segundo as diretrizes do Painel Intergovernamental sobre Mudanças Climáticas (IPCC), com base nos dados dos Inventários Brasileiros de Emissões e Remoções Antrópicas de Gases do Efeito Estufa, elaborado pelo Ministério da Ciência, Tecnologia e Inovação (MCTI), e em dados obtidos junto a relatórios governamentais, institutos, centros de pesquisa, entidades setoriais e organizações não governamentais" (SEEG, 2017).

3 “A Representação da United NationsEducational, Scientific and Cultural Organization (UNESCO) no Brasil é um escritório nacional da região da América Latina. Seu principal objetivo é auxiliar a formulação e operacionalização de políticas públicas que estejam em sintonia com as estratégias acordadas entre os Estados-membros da UNESCO. Sua atuação ocorre por intermédio de projetos de cooperação técnica em parceria com diversas instâncias governamentais e com setores da sociedade civil, à medida que seus propósitos venham a contribuir para as políticas públicas que estejam alinhadas com o desenvolvimento sustentável" (UNESCO, 2018). 
- OLS) para cada um dos cinco setores distintos: energia, agropecuária, mudança de uso na terra, resíduos e processos industriais.

Para a consecução dos objetivos supramencionados, este trabalho está organizado a partir desta introdução, na qual foi apresentada uma visão geral do cenário de pesquisa; em seguida, apresenta-se a seção 1 (Delineamento Analítico), em que se mostra a estrutura de diagnóstico, com exemplos de pesquisas semelhantes, desenvolvidas mundo afora. $\mathrm{Na}$ seção 2 (Análise das Emissões de $\mathrm{CO}_{2}$ no Brasil e em Mato Grosso do Sul), foi feito um levantamento no sentido de estreitar o entendimento acerca das emissões de $\mathrm{CO}_{2}$ no Brasil e a relação com o PIB per capita: a emissão de $\mathrm{CO}_{2}$ no Estado de Mato Grosso do Sul e a relação com o PIB per capita; e a emissão de GEE nas atividades do campo. Posteriormente, na seção 3 (Análise de Dados e Resultados), apresentam-se, respectivamente, os modelos utilizados e resultados das análises, enquanto, em suas Considerações Finais, este estudo traz uma conclusão resumida sobre o tema de pesquisa.

Espera-se que a presente análise econométrica contribua para a literatura disponível sobre externalidades entre economia e degradação ambiental, modificando e estendendo os modelos convencionais, incluindo setores e variáveis adicionais.

\section{DELINEAMENTO ANALÍTICO}

O desenvolvimento das nações depende de resultados econômicos e produtivos com capacidade para alinhar crescimento a processos sustentáveis, especialmente ambientais. No setor agropecuário, encontrase uma vasta discussão sobre produção, impactos e produtividade, pois há estreito relacionamento entre economia e produção, que se consolida pela composição cíclica de quanto maior a produção, maior é o grau de fortalecimento da economia, isto devido ao aumento da produtividade (IPEA, 2014). Na agropecuária, deve-se considerar a possibilidade de que a adoção de estratégias mais eficientes poderia refletir na maximização da produtividade e, por consequência, gerar majoração do patamar de desenvolvimento econômico.

Os estudos que relacionam desenvolvimento econômico dos países e seu alinhamento com os níveis de emissão de gás carbônico (dióxido de carbono ou $\mathrm{CO}_{2}$ ) podem ser divididos em dois momentos: no primeiro, havia uma forte correlação positiva entre os níveis de crescimento 
do Produto Nacional Bruto (PNB ou GNP, na sigla em inglês) e as emissões de dióxido de carbono. Isso sugeria que, para haver crescimento do país, a condição necessária estaria ligada ao aumento da emissão do referido gás.

Naquela primeira fase de análises, os estudos estabeleciam relações entre os possíveis fatores condicionantes de emissões do $\mathrm{CO}_{2} \mathrm{e}$ sua notabilidade em relação à degradação ambiental. Dentre tais pesquisas, destacam-se os estudos Kraft e Kraft (1978), os quais verificaram que a produção compõe uma causalidade unidirecional correlacionada ao consumo de energia e, consequentemente, emissão de dióxido de carbono, nos Estados Unidos entre 1947 e 1974. As análises destes autores se alinham a estudos como o de Ghosh (2010), que verificou, ao notar, na Índia, a causalidade de nexo entre emissões de $\mathrm{CO}_{2}$ e o desenvolvimento econômico. Na África, Akinlo (2008) seguiu esta composição, alocando a análise entre o consumo de energia e o desenvolvimento econômico. A América Central foi estudada por Apergis e Payne (2009), também com a indicação de nexos entre o consumo de energia e o desenvolvimento econômico. O Canadá foi analisado por Ghali e El-Sakka (2004), na composição de relações entre o uso da energia e a produção. Na Europa, mais especificamente na França, Ang (2007) apontou para composições semelhantes. Ainda existem estudos que consideram fatores diversos, como a renda, e, como exemplo de tais pesquisas, Coondoo e Dinda (2008) verificaram a composição entre a renda e as emissões de dióxido de carbono. Lee, em seus estudos $(2005 ; 2006)$, demonstrou a relação entre o consumo de energia e a produção por índices do Produto Interno Bruto dos países pertencentes ao G-11. Shaari et al (2017) utilizaram modelos econométricos lineares e não lineares para medir os efeitos da Pesquisa \& Desenvolvimento (P\&D) sobre as emissões de $\mathrm{CO}_{2}$. Para os trabalhos empíricos citados, assume-se como hipótese, então, a condição de que o desenvolvimento econômico traz prejuízo ao ambiente, sendo necessário o entendimento criterioso deste nexo para a construção de estratégias mitigadoras, capazes de suprir as necessidades econômicas e ambientais.

Em um segundo momento, por outro lado, as pesquisas apontaram para o seguinte: o desenvolvimento tecnológico de um país permite que haja avanço econômico, sem que isso se reflita necessariamente no aumento das emissões de dióxido de carbono, invertendo a correlação anteriormente descrita. As indicações do segundo estudo sugerem que $\mathrm{CO}_{2}$ e desenvolvimento econômico não são, obrigatoriamente, eventos mutuamente dependentes. Zhang \&Cheng (2009) mostraram, na China, os 
nexos entre consumo de energia, emissão de carbono e desenvolvimento econômico.Tiwari (2011), na Índia, examinou a causalidade na dinâmica entre consumo de energia, emissões de $\mathrm{CO}_{2}$ e crescimento econômico, concluindo acerca da necessidade de um maior esforço para explorar o uso de energias renováveis, gerando produtividade, sem retardar o desenvolvimento econômico e diminuindo emissões de $\mathrm{CO}_{2}$. Corroborando a assimilação, a Agência Internacional de Energia (AIE, 2016) confirmou o desacoplamento das emissões globais e crescimento, publicando que as emissões globais de dióxido de carbono relacionadas à energia, a maior fonte de emissões de gases do efeito estufa causadas pelo homem, permaneceram estáveis em 2014 e 2015. Estes dados mostram a tendência à desvinculação do desenvolvimento econômico como um dos principais colaboradores do aquecimento global.

Identificar o estágio de desenvolvimento tecnológico de um país, ou de uma região, pode oferecer evidências que permitam inferir sobre a maior ou menor correlação entre a economia e a elevação dos níveis de carbono na atmosfera.

\section{ANÁLISE DAS EMISSÕES DE CO2 NO BRASIL E EM MATO GROSSO DO SUL}

No âmbito da Contribuição Nacional Determinada (intended Nationally Determined Contribution - iNDC) $)^{4}$ o Brasil assinou o tratado do clima, comprometendo-se com a redução de $37 \%$ das emissões de $\mathrm{CO}_{2}$ para 2025 e de 43\% para 2030, comparadas às emissões de 2005 (MMA, 2016). Podem ser somados a esta meta compromissos de garantir $45 \%$ de fontes renováveis na matriz energética, com a ampliação de $23 \%$ das fontes renováveis para fornecimento elétrico e a ambição de erradicação do desmatamento ilegal (MMA, 2015). O governo brasileiro tem apontado, ainda, para uma redução de 41,1\% nas emissões de GEE entre 2005 e 2012. E, ainda nos termos das informações governamentais, a principal ação para o alcance deste valor está em uma política agressiva de controle do desmatamento da Amazônia Legal, que abrange o estabelecimento de fronteiras agrícolas, englobando demarcação e obediência quanto a áreas de proteção ambiental, utilizando o indicador mudança do uso da terra e da floresta (MMA, 2015). Neste sentido, e de acordo com Kässmayer e Neto 4 http://www.mma.gov.br/images/arquivo/80108/BRASIL\%20iNDC\%20 portugues\%20FINAL.pdf 
$(2016)^{5}$, o Acordo de Paris, ratificado pelo Brasil em 2016, representa o início de um novo paradigma de sustentabilidade: o paradigma climático, cuja incitação é a de harmonizar a política climática com o desenvolvimento sustentável em todos os setores.

Os aportes de produção, agronegócio e emissões de $\mathrm{CO}_{2}$ compõem um grupo maior de objetivos ligados às ciências ambientais. É grande o volume de pesquisas, abordando: as relações entre os fatores de produção e do agronegócio, os de produção e os de emissões de $\mathrm{CO}_{2}$, assim como os do agronegócio e os de emissões de dióxido de carbono. Estes estudos focam, principalmente, em uma análise isolada dos fatores, enquanto a proposição do presente trabalho é analisar os fatores compondo uma inter-relação entre eles.

O Brasil se apresenta como uma das grandes forças do mercado mundial, chegando a compor indicadores de liderança em vários setores de produção, como é o caso do agronegócio, no qual estão produtos como a soja, o milho, as carnes, entre outros. Assumindo características dos países em desenvolvimento, observam-se aumentos absolutos dos números de habitantes, de consumo e de pressão sobre fronteiras, que são imposições da economia globalizada (Gasques, et al, 2014). Esta composição tão específica condiciona um estudo transversal, entre um determinado recorte histórico, por meio do qual resultados e observações da relação das variáveis devem ser colhidos especificamente para o Brasil.

Todavia, sendo o Brasil um país em desenvolvimento e, assim, tendo seu arcabouço tecnológico em igual situação, seria possível ao país cumprir os objetivos dos acordos sobre o clima, como foi o referido Acordo de Paris? O mesmo cenário poderia ser replicado ao estado de Mato Grosso do Sul? Para se responder a tais questões, serão descritos os níveis de emissão de GEE e sua comparação com o Produto Interno Bruto per capita, tanto em âmbito nacional quanto estadual. O período escolhido se inicia em 2002, por conta de ter havido a Rio+10, marco relevante para o desenvolvimento sustentável, finalizando a série histórica no ano mais próximo possível da realização do presente estudo, ou seja, gerando análises apoiadas nos dados mais recentes disponíveis. Portanto, os dados de emissão de $\mathrm{CO}_{2}$ e PIB per capita no Brasil abrangem Produto Interno Bruto e emissões até dezembro de 2016, enquanto os dados disponíveis para o PIB per capita do Mato Grosso do Sul contam com dados atualizados até dezembro de 2015.

5https://www12.senado.leg.br/publicacoes/estudos-legislativos/tipos-de-estudos/textospara-discussao/td215 


\subsection{Emissões de CO2 no Brasil e a Relação com o PIB per capita}

Apresentam-se, a seguir, gráficos que indicam o comportamento das emissões totais de dióxido de carbono no Brasil, de 2002 a 2016.

\section{Quadro 1: Emissões Totais de CO2 no Brasil por Setor (2002-2016)}
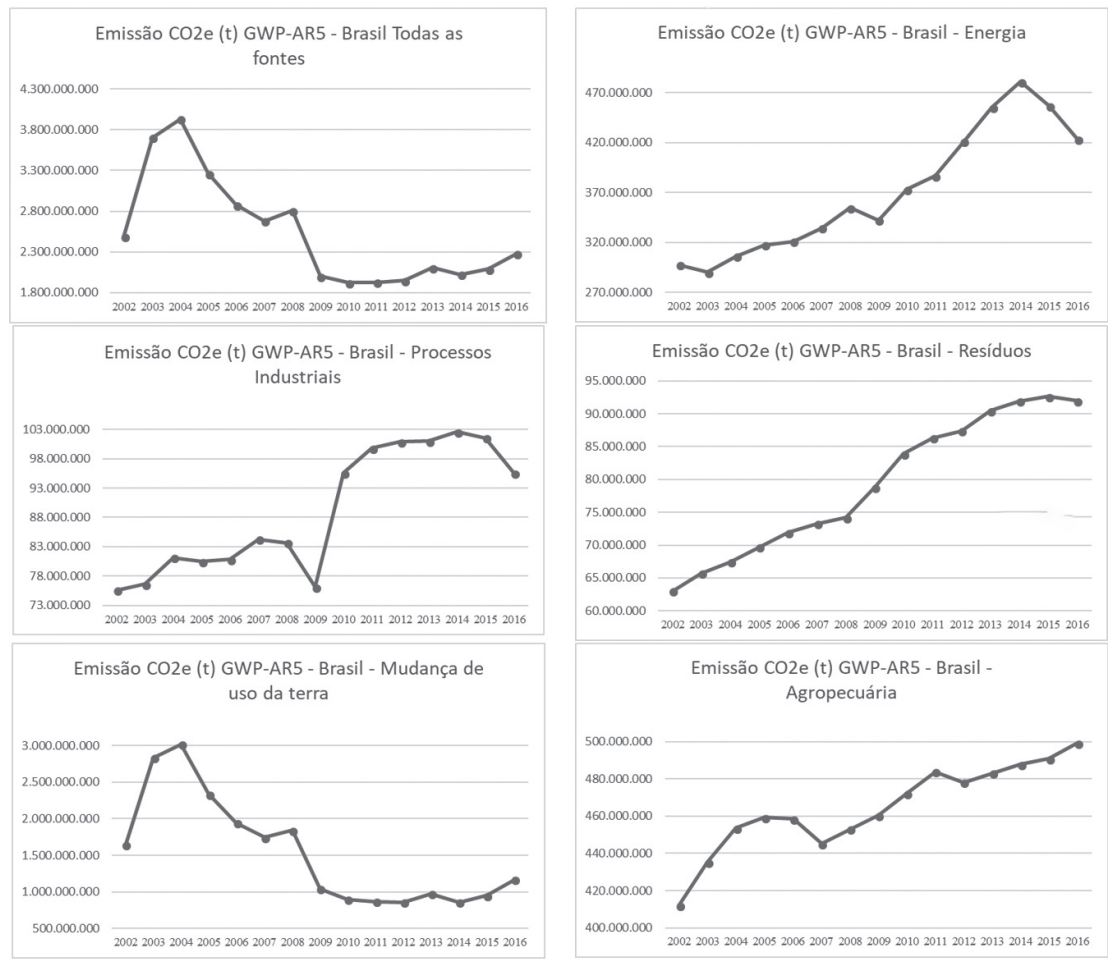

Fonte: SEEG (2017).

O Quadro 1 contém a curva de emissão de $\mathrm{CO}_{2}$ no Brasil, agregando todas as fontes, ou setores da economia. Deixa evidente que, após um rápido período de aumento, que abrange o período entre 2002 e 2004, há uma consistente curva descendente na emissão do gás, até sua relativa estabilização, entre os anos de 2010 e 2012. Ao final daquele ano, há o início de um movimento ascendente, representando aumento na quantidade de toneladas de $\mathrm{CO}_{2}$ na atmosfera, até o final da série, no ano de 2016. Ainda assim, os valores registrados entre 2010 e 2016 ficam muito abaixo daqueles registrados ao longo da década anterior.

Quando confrontados, os dadosindicam que apenas oAgronegócio 
e a Mudança no Uso da terra foram os setores que influenciaram a elevação de emissões durante todo o período considerado, ao passo que os demais setores - Energia, Processos Industriais e Resíduos - acompanharam o movimento de alta apenas entre os anos de 2010 e 2014. Isso posto, é válido concluir que as duas primeiras fontes citadas - Agronegócio e Mudança no Uso da terra - determinaram a inflexão da tendência de queda, notadamente a partir de 2014. Assim, se, de um modo geral, as emissões caíram entre 2002 e 2016, há alguma pressão de alta nos três anos finais da série.

No entanto, é prudente considerar que o período 2013-2016 ainda seja muito curto para confirmar uma efetiva inversão de tendência - o que poderá ser avaliado em estudos subsequentes. Somente a Agropecuária apresentou crescimento nas emissões de dióxido de carbono durante todo o recorte histórico.

O PIB per capita, cujo movimento está indicado no Quadro 2, apresentou-se invariavelmente em alta em todo o momento histórico considerado.

\section{Quadro 2: PIB per capita no Brasil, de 2002 até 2015}

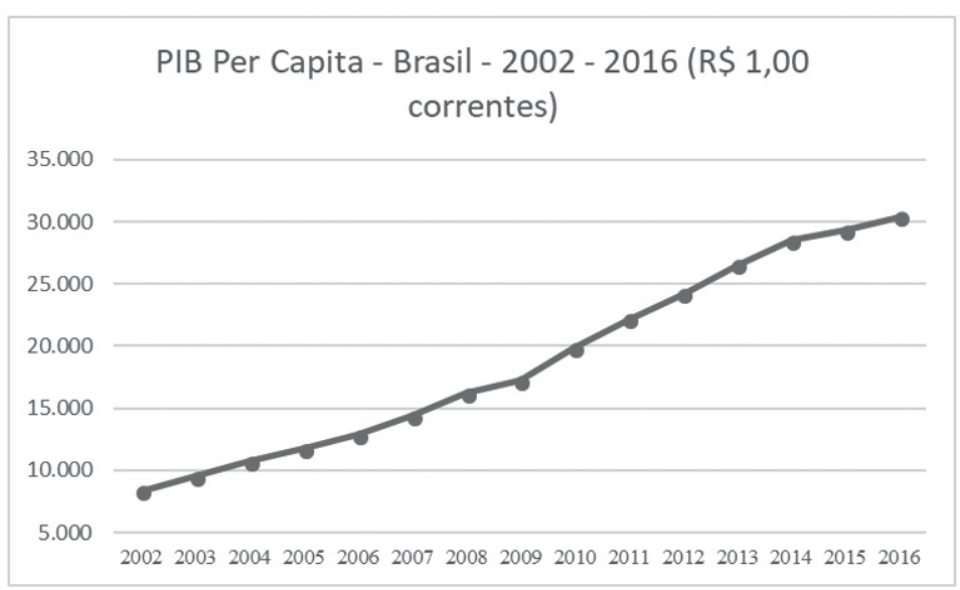

Fonte: SGS (2018).

A conclusão é que, ainda que tenha havido desenvolvimento econômico registrado no Brasil pelo indicador que mostra a riqueza produzida, dividida entre a população do país, registrou-se a redução na emissão do principal GEE - permitindo estabelecer uma relação 
inversamente proporcional entre geração de riqueza e despejo de gás $\mathrm{CO}_{2}$ na atmosfera.

\subsection{Emissões de CO2 no Mato Grosso do Sul e a Relação com o PIB per capita}

O estado do Mato Grosso do Sul, amparado pela Lei Estadual 4.555/20146 (ESTADO DE MATO GROSSO DO SUL), assumiu uma redução voluntária nas emissões de gases de efeito estufa de $20 \%$ até 2020 , em comparação com os lançamentos de GEE de 2005. Para alcançar este objetivo, o legislador designou várias estratégias, incluindo esta unidade da federação no Programa sobre Mudança Climática (PROCLIMA), que envolve uma série de ações e compromissos estaduais para mitigar as emissões de gases causadores de efeito estufa. Uma dessas ações é o Terra Boa, cujo objetivo é recuperar dois milhões de hectares de áreas degradadas no Mato Grosso do Sul, equivalentes a 13\% do compromisso (15 milhões de hectares) que o Brasil assumiu na COP 21(IMASUL/2016).

Em um ato contínuo, o Projeto Estado Carbono Neutro foi parte integrante do PROCLIMA, realizado pela Secretaria de Estado de Meio Ambiente e Desenvolvimento Econômico (SEMADE), cujo propósito é gerar as bases metodológicas para uma economia de baixo carbono no Mato Grosso do Sul, desenvolvendo e adaptando tecnologias para a redução e mitigação das emissões de gases de efeito estufa nos diversos setores da economia do estado (SEMADE/2016).

Assim como se procedeu em relação às conjecturas sobre as possibilidades de o Brasil alcançar o que foi proposto pelos acordos internacionais firmados, é igualmente necessário avaliar o potencial de que o MS cumpra suas próprias metas relacionadas ao clima, e os gráficos a seguir mostram como se comportou esta unidade da federação no decorrer do mesmo recorte histórico dos dados apresentados para o Brasil. A

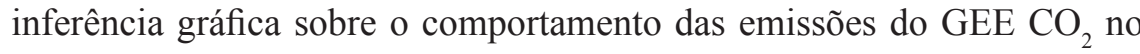
estado de Mato Grosso do Sul buscou identificar o grau de aderência entre o cenário deste local e o do país. Desta feita, consideram-se os movimentos descritos no Quadro 3, abaixo.

6 A Lei Estadual n 4.555 , de 15 de julho de 2014, instituiu a Política Estadual de Mudanças Climáticas (PEMC), no âmbito do Território do Estado de Mato Grosso do Sul. 


\section{Quadro 3 \\ Emissões Totais de CO2 no Mato Grosso do Sul por Setor}

(2002-2016).
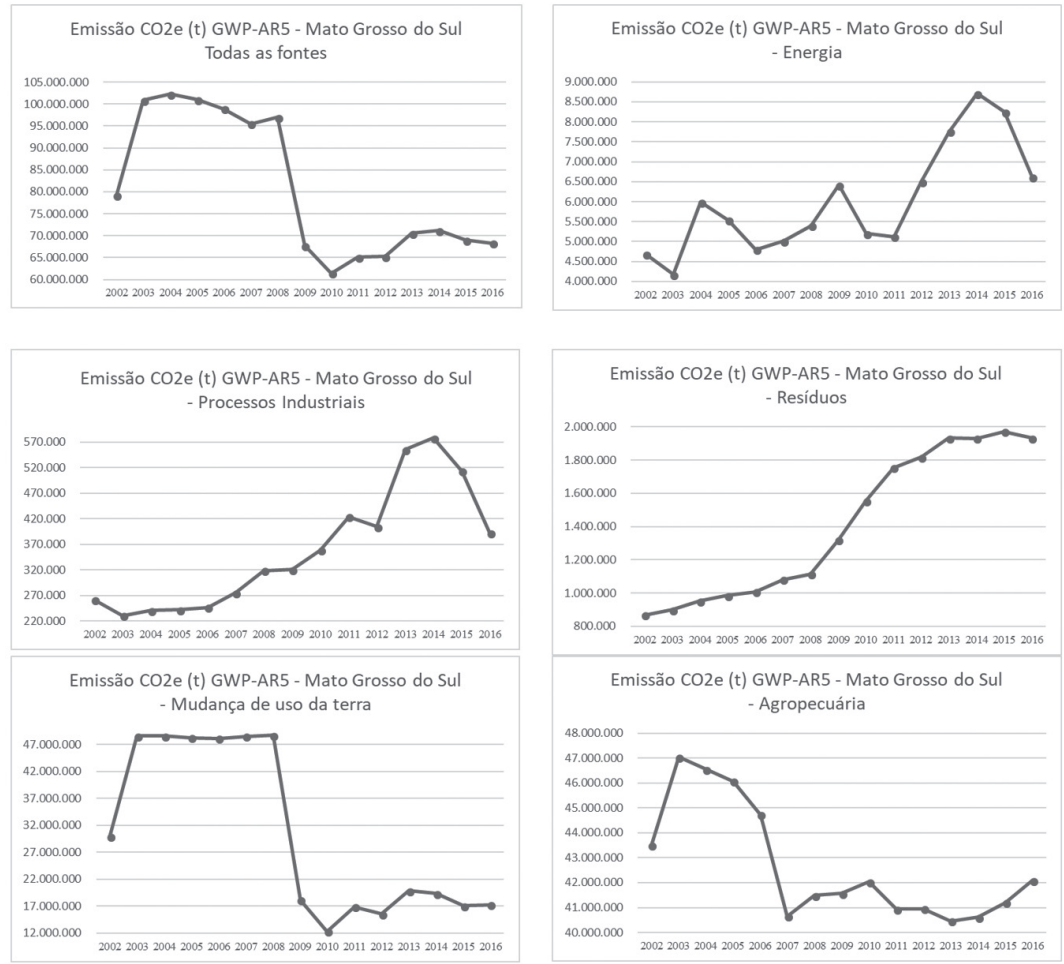

Fonte: SEEG, 2017.

O quadro geral aponta para uma equivalência direta entre o crescimento na emissão do gás no MS e no Brasil, entre 2002 e 2004. A partir daí, enquanto houve rápido declínio no país entre 2004 e 2007, o Mato grosso do Sul registrou índices constantemente altos até 2008. A partir daquele ano, iniciou-se um forte declínio, culminando com o nível mais baixo de toda a série em 2010 .

O despejo de $\mathrm{CO}_{2}$ no estado de MS operou na contramão do que ocorreu no Brasil entre todo o restante dos registros: se, de 2010 até 2014, houve aumento nas emissões locais, iniciou-se um movimento de baixa nas emissões a partir deste último até o final da série, em 2016.

Quando consideradas todas as fontes de emissão de $\mathrm{CO}_{2}$ no 
MS, nota-se que os setores de energia, processos industriais, resíduos e mudança de uso da terra se comportam de modo semelhante ao movimento nacional, com pequenas variações entre registros anuais - mas preservando o movimento geral. Destaque se faz ao comportamento das emissões na agropecuária: a curva de alta é bastante clara no Brasil; já no MS, o panorama geral é de queda, com inflexão para o crescimento notado apenas a partir de 2013.

Idêntico comportamento é registrado para o PIB per capita que, no estado de Mato Grosso do Sul, mostra clara aderência ao movimento deste indicador em âmbito nacional (Quadro 4).

\section{Quadro 4}

Produto Interno Bruto per capita no Mato Grosso do Sul, de 2002 até 2015

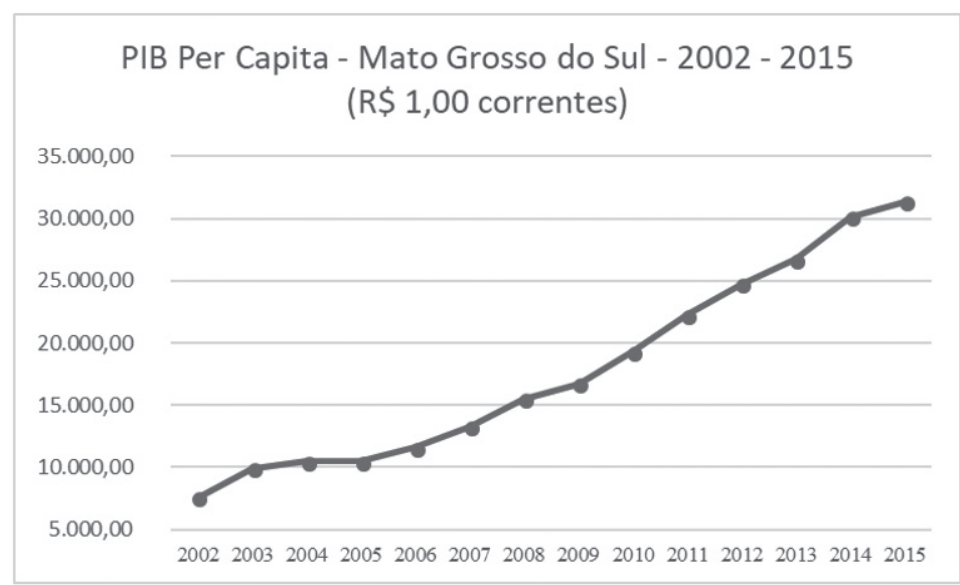

Fonte: SGS (2018).

Os dados até aqui apresentados indicam que há desenvolvimento econômico coincidente no Brasil e no estado de Mato Grosso do Sul com a também congruência de comportamento declinante na emissão de gás carbônico na atmosfera. A dúvida que se forma, no entanto, diz respeito às possíveis razões de haver maior emissão de $\mathrm{CO}_{2}$ nas atividades relacionadas à agropecuária e à mudança de uso da terra - o que será discutido a seguir. 


\subsection{Emissão de GEE nas Atividades do Campo}

A primeira fase deste estudo buscou entender a dinâmica de emissões de GEE, baseadas em inventários disponíveis e sua relação com mudanças no uso da terra e atividades agrícolas no Brasil e no Mato Grosso do Sul. Os inventários atuais são baseados em padrões globais e nacionais, fatores de emissão que não estão completamente adaptados a determinadas características do estado, sendo que alguns fatores de emissão estão, provavelmente, superestimando as emissões líquidas de GEE.

O que pode explicar o aumento na emissão de GEE nas atividades ligadas ao campo é que a conversão de áreas de florestas, cerrados e campos para áreas agrícolas ou pastagem diminui o teor de matéria orgânica nos solos tropicais e subtropicais devido às consequências de curto e longo prazos dos distúrbios ocasionados pelas operações de preparo do solo, associados aos baixos níveis de adição de material orgânico (SARTORI et al., 2006). O processo de perda de carbono do solo para a atmosfera é chamado de emissão de $\mathrm{CO}_{2}$ do solo $\left(\mathrm{FCO}_{2}\right)$, ou respiração do solo, resultante da atividade microbiana e respiração das raízes, sendo considerada a segunda maior fonte de dióxido de carbono para a atmosfera, perdendo apenas para os oceanos. A respiração do solo é determinada por um conjunto de fatores, tais como a temperatura e a umidade do solo (EPRON et al., 2004). No processo de emissão de $\mathrm{CO}_{2}$, particularmente, o transporte do gás do interior do solo até a superfície é governado pela equação de difusão, que, por sua vez, é influenciada, em primeira ordem, por alterações na temperatura e umidade do solo (KANG et al., 2003).

Em 2014, os 7,74 milhões de hectares de árvores plantadas no Brasil foram responsáveis pelo estoque de aproximadamente 1,69 bilhão de toneladas de dióxido de carbono $\left(\mathrm{t} \mathrm{CO}_{2}\right)$, representando um incremento de 1,2\% em relação a 2013. Os plantios de eucalipto ocupam 5,56 milhões de hectares da área de árvores plantadas no Brasil, o que representa 71,9\% do total, localizados principalmente nos estados de Minas Gerais $(25,2 \%)$, São Paulo (17,6\%) e Mato Grosso do Sul 14,5\% ou 803.699 hectares (IBÁ, 2015). De acordo com alguns estudos, o eucalipto tem maior potencial para estocar carbono atmosférico na biomassa aérea e no solo, principalmente quando associado à pastagem ou culturas anuais e, especialmente, na conversão de terras degradadas em terras produtivas e de fontes de energia renováveis (LA SCALA et al., 2012).

A mudança do uso do solo causada pela conversão de florestas em 
pastagens ou em áreas agrícolas modifica a cobertura e, consequentemente, o conteúdo de carbono no solo. $\mathrm{O}$ equilíbrio entre o carbono retido e o perdido no solo é afetado pela mudança do uso do solo até que um novo "equilíbrio" seja retomado (GUO; GIFFORD, 2002).

Um conjunto de medidas de contenção de desmatamento no Brasil, principalmente na Amazônia, reduziu, nos 23 anos compreendidos entre 1990 e 2012, 56\% do total de emissões de toneladas de carbono equivalente $\left(\mathrm{t} \mathrm{CO}_{2} \mathrm{e}\right)$. Em 1990, as emissões brutas correspondiam a 1,25 bilhões de toneladas de $\mathrm{CO}_{2}$, atingindo, em 2004, um pico de dois bilhões de $\mathrm{C}_{2} \mathrm{e}$. Nos anos seguintes, iniciou-se um processo de redução do desmatamento, que diminuiu as emissões pela metade, em 2007, atingindo

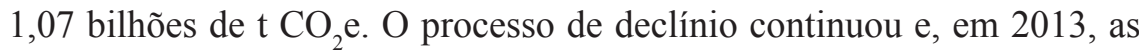
emissões por mudanças de uso do solo atingiram o seu menor nível, com

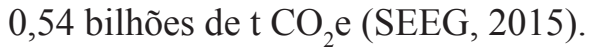

Estudos anteriores podem confirmar o potencial de redução de emissão de carbono associando ao plantio direto. No Relatório de Síntese Técnica, Uso da Terra, Mudanças do Uso da Terra e Florestas, realizado em 2010, já se previa que, no cenário de baixo carbono, $100 \%$ da área de produção de algodão, arroz, feijão, milho e soja seriam convertidos em plantio direto, alcançando destaque em 2015. Todavia, observou-se uma queda imediata, confirmada no gráfico, logo após 2009. O relatório ainda conceitua o uso do sistema de plantio direto como preconizador de três ações básicas para a sustentabilidade do sistema: o plantio deveria ser feito continuamente na forma direta, sem o revolvimento tradicional do solo; foram utilizadas culturas capazes de deixar alta qualidade de palha para manter o solo coberto com resíduos por todo ano; e, por fim, o uso de rotação de culturas, no verão e inverno, seria necessário para quebrar ciclos de pragas e doenças e melhorar a reciclagem de nutrientes do solo.

\section{ANÁLISE DOS DADOS E RESULTADOS}

A presente pesquisa se qualifica como quantitativa, de caráter exploratório, com análise empírica, utilizando abordagem econométrica, seguindo os procedimentos de Ang (2007), Gosh (2010), Borhan, Ahmed e Hitam (2011) e Shaari et al (2017).

Os dados apreciados neste trabalho foram obtidos de fontes 
secundárias, alocados em séries temporais, e abrangem o período entre 2002 e 2016. Para dados específicos das emissões de dióxido de carbono, foi utilizada a base de dados da SEEG, gerados a partir de diretrizes do Painel Intergovernamental sobre Mudanças Climáticas. Os dados de caráter econômico foram obtidos nas bases de dados do Banco Central do Brasil (BACEN).

\subsection{Método de Estimação}

Para análise empírica, foi definida a modelagem econométrica como procedimento metodológico, e o método para a estimação foi o de Mínimos Quadrados Ordinários (MQO). A partir de cinco modelos teóricos, o objetivo é analisar a relação entre as variáveis de crescimento econômico e as emissões de $\mathrm{CO}_{2}$. As variáveis explicativas (independentes) foram o PIB per capita e PIB per capita ao quadrado; para analisar a teoria do "U" invertido, todos os dados foram loglinearizados. Já para as emissões de dióxido de carbono, houve uma divisão em cinco setores distintos: energia, agropecuária, mudança de uso na terra, resíduos e processos industriais

A qualidade e ajustes dos valores obtidos na regressão são medidos com o índice " $\mathrm{R}^{2 "}$ (R-squared). O coeficiente de determinação, chamado de $\mathrm{R}^{2}$, é uma medida de ajustamento de um modelo estatístico linear generalizado, como a regressão linear, em relação aos valores observados. $\mathrm{O} \mathrm{R}^{2}$ varia entre 0 e 1 , indicando, em percentagem, o quanto o modelo consegue explicar os valores observados. Quanto maior o $\mathrm{R}^{2}$, mais explicativo é o modelo; ou seja, quanto melhor o modelo, mais se ajusta à amostra (GUJARATI, 2011).

\subsection{Modelos Teóricos}

\subsection{Modelo 1}

$$
\text { CO2terra }_{t}=\beta_{0}+\beta_{1} P I B p c_{t}+\beta_{2} P I B p c_{t}^{2}+\varepsilon_{t}
$$

Em que são os dados de emissão do setor mudança de uso na terra, e as variáveis explicativas são Produto Interno Bruto total (PIB), PIB per capita (PIBpc), PIB do agronegócio (PIBAgro) e área plantada (Área), em hectares. Os “" são os coeficientes angulares da regressão linear e é o erro aleatório. 


\subsubsection{Modelo 2}

$$
\text { CO2resíduo }_{t}=\beta_{0}+\beta_{1} \operatorname{PIBpc}_{t}+\beta_{2} \operatorname{PIBpc}_{t}^{2}+\varepsilon_{t}
$$

Em que " são os dados de emissão do setor de resíduos. As variáveis explicativas para crescimento econômico são as mesmas nos cinco modelos.

\subsubsection{Modelo 3}

$$
\text { CO2indústria }{ }_{t}=\beta_{0}+\beta_{1} P I B p c_{t}+\beta_{2} P I B p c_{t}^{2}+\varepsilon_{t}
$$

Em que " é a variável dependente que caracteriza os dados das emissões de dióxido de carbono pelo setor de processos industriais.

\subsubsection{Modelo 4}

$$
\text { CO2agronegócio }_{t}=\beta_{0}+\beta_{1} P I B p c_{t}+\beta_{2} P I B p c_{t}^{2}+\varepsilon_{t}
$$

Em que é a variável dependente que caracteriza os dados das emissões de dióxido de carbono pelo setor de agronegócios.

\subsubsection{Modelo 5}

$$
\text { CO2energia }_{t}=\beta_{0}+\beta_{1} P I B p c_{t}+\beta_{2} P I B p c_{t}^{2}+\varepsilon_{t}
$$

Em que é a variável dependente que caracteriza os dados das emissões de dióxido de carbono pelo setor de energia.

Com a construção teórica dos modelos, este estudo empírico utilizou o software " $R$ " para estimar os valores preditos, a partir de regressão linear.

7 R version 3.4 .3 (2017-11-30) -- "Kite-EatingTree", Copyright (C) 2017 The R Foundation for StatisticalComputing. https://www.r-project.org/ 


\subsection{Resultados}

Os estudos aplicados na área de crescimento econômico e emissões de $\mathrm{CO}_{2}$ tratam de modelos econométricos baseados na curva de Kuznets, que analisam a variação marginal da poluição na produtividade e renda da população. Assim como em Akinlo (2008), Zhang \&Cheng (2009), Ghosh (2010), Borhan et al, (2012) e Shaari et al (2017). Nesta pesquisa, utilizou-se regressão linear de cinco modelos distintos, um para cada setor econômico.

Uma simples correlação entre o total de emissões de $\mathrm{CO}_{2}$ e o PIB para o Mato Grosso do Sul entre 2002 e 2016 é apresentada no Gráfico 1.

\section{Gráfico 1}

\section{Correlação Linear entre PIB per capita e Emissões Totais de CO2 no MS(2002 a 2016)}

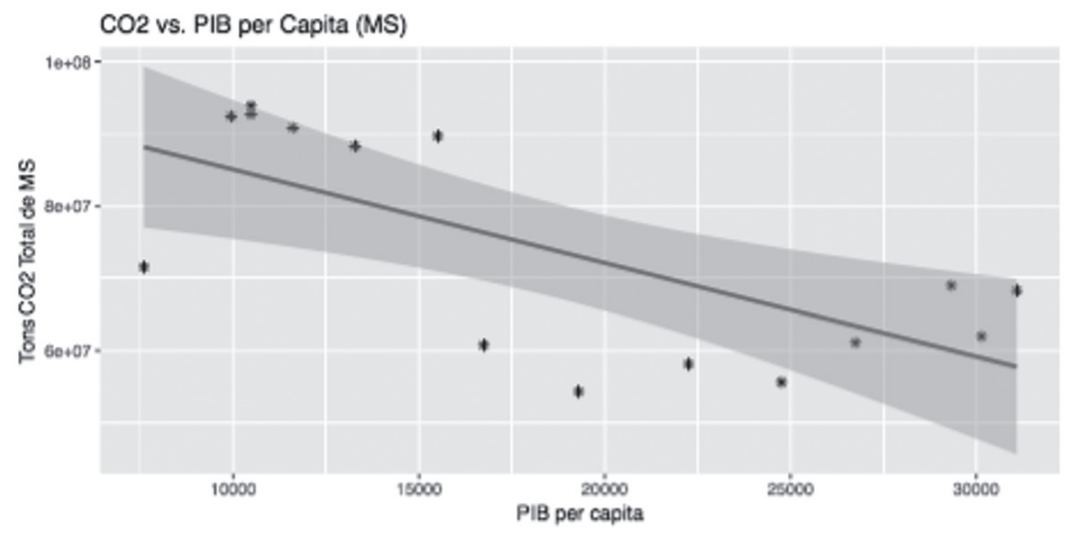

Fonte: BACEN e SEEG (2017).

A correlação linear entre o crescimento do PIB e as emissões de $\mathrm{CO}_{2}$ no Mato Grosso do Sul mostra uma relação inversa, pois, enquanto a economia cresce, as emissões diminuem, em contraste com os estudos de países desenvolvidos como China, EUA e França (Ang, 2008; Ghosh, 2010). Essa correlação traz evidências iniciais de produção de energia limpa (uso de hidrelétricas), além da adoção de tecnologias avançadas para aumento da produtividade na agropecuária nacional ${ }^{8}$.

$\mathrm{Na}$ Ásia, Borhan et al (2012) e Shaari et al (2017) mostram

8 Ver: GASQUES, J. G.; CONCEIÇÃO, J. C. P. R. Transformações estruturais da agricultura e produtividade total dos fatores. Texto para Discussão n. 768. Brasília: Ipea, 2000. 
evidências de que há uma correlação positiva entre o crescimento econômico e as emissões de $\mathrm{CO}_{2}$, principalmente no setor de geração de energia.

Decompondo as emissões de Mato Grosso do Sul, é possível entender melhor esse fenômeno e, para atingir esse resultado, foram estimados os modelos apresentados na Tabela 1, que mostram os resultados das estimações dos cinco modelos propostos, englobando os setores econômicos que emitem dióxido de carbono.

Tabela 1

Resultados das Estimações dos Modelos Econométricos

\begin{tabular}{|c|c|c|c|c|c|}
\hline & \multicolumn{5}{|c|}{ Dependent variable: } \\
\hline & $\begin{array}{c}\text { Energia } \\
(1)\end{array}$ & $\begin{array}{c}\text { Agro } \\
(2)\end{array}$ & $\begin{array}{c}\text { ProcInd } \\
\text { (3) }\end{array}$ & $\begin{array}{c}\text { Res } \\
(4)\end{array}$ & $\begin{array}{c}\text { Mut } \\
(5)\end{array}$ \\
\hline PIBpc & $\begin{array}{l}-5.217 \\
(4.205)\end{array}$ & $\begin{array}{l}-3.638 \\
(2.080)\end{array}$ & $\begin{array}{l}-4.829 \\
(3.719)\end{array}$ & $\begin{array}{c}-4.439^{*} \\
(2.221)\end{array}$ & $\begin{array}{c}5.925 \\
(11.361)\end{array}$ \\
\hline Pibpc2 & $\begin{array}{c}0.287 \\
(0.217)\end{array}$ & $\begin{array}{c}0.187 \\
(0.107)\end{array}$ & $\begin{array}{c}0.281 \\
(0.192)\end{array}$ & $\begin{array}{c}0.262^{* *} \\
(0.114)\end{array}$ & $\begin{array}{l}-0.354 \\
(0.585)\end{array}$ \\
\hline Constant & $\begin{array}{l}39.083^{*} \\
(20.372)\end{array}$ & $\begin{array}{c}35.069^{* * *} \\
(10.076)\end{array}$ & $\begin{array}{c}33.088^{*} \\
(18.018)\end{array}$ & $\begin{array}{l}32.286^{* *} \\
(10.758)\end{array}$ & $\begin{array}{c}-7.026 \\
(55.039)\end{array}$ \\
\hline Observations & 15 & 15 & 15 & 15 & 15 \\
\hline $\mathrm{R}^{2}$ & 0.656 & 0.205 & 0.872 & 0.954 & 0.625 \\
\hline Adjusted $\mathrm{R}^{2}$ & 0.599 & 0.072 & 0.851 & 0.946 & 0.562 \\
\hline Residual Std. Error $(\mathrm{df}=12)$ & 0.137 & 0.068 & 0.121 & 0.072 & 0.370 \\
\hline F Statistic $(\mathrm{df}=2 ; 12)$ & $11.446^{* * *}$ & 1.544 & $40.831^{* * *}$ & $123.849^{* * *}$ & $10.000^{* * * *}$ \\
\hline
\end{tabular}

A análise econométrica dos resultados da Tabela 1 permite, no caso em peculiar do setor de resíduos, percebê-lo como único modelo que apresentou significância estatística, porém, contrariando a Curva de Kuznets, no cenário de Mato Grosso do Sul. O Modelo 4 apresenta evidências de que o aumento da renda per capita diminui as emissões de $\mathrm{CO}_{2}$, e, na forma quadrática, as emissões aumentam, formando a trajetória de "U". O modelo tem ótimo ajuste com $\mathrm{R}^{2}$ de 0,954 . O resultado empírico mostra que existe um nível ótimo de renda, para o menor nível de emissões de dióxido de carbono.

Os modelos e os dados apresentam evidência empírica de desaceleração da emissão de $\mathrm{CO}_{2}$ no estado de Mato Grosso do Sul, resultante 
das medidas adotadas e já citadas e um desenvolvimento econômico em ascensão, com o provável uso de tecnologias mais sustentáveis para o aumento da produtividade e geração de energia.

\section{CONSIDERAÇÕES FINAIS}

O presente estudo foi motivado pela questão de pesquisa acerca da existência, ou não, de desenvolvimento econômico no Brasil e no estado de Mato Grosso do Sul, com a manutenção dos baluartes de sustentabilidade pertinentes aos documentos e acordos globais. A resposta poderia indicar o maior, ou menor, alinhamento do Brasil e do estado em análise aos parâmetros de desenvolvimento sustentável praticados no mundo, indicando o potencial produtivo para o desenvolvimento local.

Para que esta interrogação fosse sanada, optou-se por seguir o preconizado em recentes estudos que pudessem relacionar indicadores de desenvolvimento econômico àqueles de impactos no meio ambiente. A escolha recaiu sobre o volume de emissão de dióxido de carbono importante gás de efeito estufa - e sua comparação com o PIB per capita - medida relevante do avanço econômico por mensurar a produção e distribuição média da riqueza em um lugar. Como recorte histórico, ficou definido que o período deveria abranger desde um importante marco no desenvolvimento sustentável - que impusesse medidas mais severas no controle à emissão de poluentes - até a data em que houvesse dados mais recentes disponíveis à época da elaboração deste estudo.

Ao final, ficou constatado que, tanto o Brasil quanto o estado de Mato Grosso do Sul, realizaram, no período considerado, desenvolvimento econômico associado à baixa emissão relativa de gás carbônico. No entanto, a análise dos dados deixa uma grande questão em aberto: seriam o Brasil, no geral, e o estado do Mato Grosso do Sul, em particular, dotados de tal desenvolvimento técnico e tecnológico que lhes permitiria atingir crescimento econômico com baixa emissão de dióxido de carbono, ou haveria alguma outra excepcionalidade que permitisse tal correção? Este pode ser um tema de exploração para pesquisas posteriores.

Ainda sugere o presente estudo que, para mitigar a emissão de $\mathrm{CO}_{2}$ e reduzir a contribuição da agropecuária na emissão de gases do efeito estufa, as estratégias mais eficientes consistiriam na diminuição da queima de combustíveis fósseis, minimização do desmatamento e queimadas, manejo adequado do solo, plantio de espécies favoráveis à rápida 
incorporação de carbono excedente - como o eucalipto, por exemplo conseguindo, por fim, a maximização do sequestro de carbono, mesmo nas atividades produtivas mais intensivas, fato presente na unidade da federação estudada.

\section{REFERÊNCIAS}

AKINLO, A.E. Energy consumption and economic growth: Evidence from 11 Sub-Sahara African countries. In: Energy Economics, Elsevier, vol. 30(5), pages 2391-2400, 2008. Disponível em: $<$ https://www.sciencedirect. com/science/article/pii/S014098830800025X>. Acesso em: 06.mai.2018.

ANG, J.B. $\mathrm{CO}_{2}$ emissions, energy consumption, and output in France. In: Energy policy. Vol.35, pages. 4772-4778, 2007. Disponível em: <https:// www.sciencedirect.com/science/article/pii/S0301421507001498>. Acesso em: 02.mai.2018.

APERGIS, N.; PAYNE, J. Energy consumption and economic growth in Central America: Evidence from a panel cointegration and error correction model. In: EconPapers, Economics at yourf inger tips. 2009. Disponível em: <https://econpapers.repec.org/article/eeeeneeco/ v_3a31_3ay_3a2009_3ai_3a2_3ap_3a211-216.htm>. Acesso em: 02.mai.2018.

\section{BANCO CENTRAL DO BRASIL (BACEN)}

BANCO INTERNACIONAL PARA RECONSTRUÇÃO E DESENVOLVIMENTO/BANCO MUNDIAL. Estudo de baixo carbono para o Brasil. Relatório de Síntese Técnica. Uso da Terra, Mudanças do uso da Terra e Florestas. 2010. Disponivel em: $<\underline{\text { http://siteresources.worldbank. }}$ org/BRAZILINPOREXTN/Resources/3817166-1276778791019/ UsoTerra_Final_Portugue.pdf $>$. Acesso em: 11.maio.2018.

BORHAN, H.; AHMED, E.M.; HITAM, M. The Impactof $\mathrm{CO}_{2}$ onEconomicGrowth in Asean 8. In: SciVerseScienceDirect, 2012. Disponível em: $<$ https://ac.els- cdn.com/S1877042812004156/1-s2.0S1877042812004156-main.pdf?_tid=d59356d3-ae00-4861-be2d-142db fb47533\&acdnat $=1525228406 \_616 b 3 e 920$ fae 1a9fb346576903aebd88>. Acesso em: 01.maio.2018. 
Chien-Chiang Lee. The CausalityRelationshipbetween Energy Consumptionand GDP in G-11 Countries Revisited. Energy Policy, 34, no. 4. UK: Elsevier, 2006.

Chien-Chiang Lee. The CausalityRelationshipbetween Energy Consumptionand GDP in G-11 Countries Revisited. Energy Policy, 34, no. 4. UK: Elsevier, 2006.

COONDOO, D.; DINDA, S. Carbondioxideemissionandincome: A temporal analysisof cross-country distgributionalpatterns. In: ScienceDirect, Elsevier, 2008. Disponível em: $<$ https://pt.scribd.com/ document/242572213/Coondoo-and-Dinda-2008-pdf $>$. Acesso em: 03.mai.2018.

EPRON, D.; NOUVELLON, Y.; ROUPSARD, O.; MOUVONDY, W.; MABIALA, A.; SAINT-ANDRÉ, L.; JOFFRE, R. JOURDAN, C.; BONNEFOND J. M.; BERBIGIER, P.; HAMEL, O. Spatialand temporal variationsofsoilrespiration in a Eucalyptus plantation in Congo. Forest Ecologyand Management, Amsterdam, v. 202, n. 1-3, p. 149-160, 2004.

ESTADO DE MATO GROSSO DO SUL. Lei $\mathrm{n}^{\circ} 4.555$, de 15 de julho de 2014. Disponível em $<$ http://aacpdappls.net.ms.gov.br/appls/legislacao/ secoge/govato.nsf/448b683bce4ca84704256c0b00651e9d/c8e1c43dcb65a 53104257d170051d5b1?OpenDocument>. Acesso em 14.mai.2018.

GASQUES, J.G.; BASTOS, E.T.; VALDES,G.; BACCHI, M.R.P.; Produtividade da agricultura. Resultados para o Brasil e estados selecionados. In Revista de Política Agrícola. Ano XXIII, n³, jul./ago./ set.2014. Disponível em: https://www.alice.cnptia.embrapa.br/alice/ bitstream/doc/1003973/1/Produtividadedaagricultura.pdf. Acesso em: 08.abr.2018.

GHALI, K.H.; EL-SAKKA, M. Energy use and output growthin Canada: a multivariatecointegrationanalysis. In: EconPapers, Economicsatyourfingertips, 2004. Disponível em: <https://www. sciencedirect.com/science/article/pii/S0140988303000562>. Acesso em: 02.maio.2018.

GUJARATI E PORTER; Análise de Regressão Linear Múltipla . Instituto de Ensino e Pesquisa. Capítulo 7 e 8, 2011. 
GUO L. B; GIFFORD, R. M. Soilcarbon stocks andland use change: a meta analysis. Global ChangeBiology, Chichester, v. 8, p. 345-360, 2002.

INDÚSTRIA BRASILEIRA DE ÁRVORES - IBÁ. Anuario estatístico da IBA: ano base 2014- 2015, 2015. $80 \mathrm{p}$

INFOAGRO. Balanço anual do agronegócio sul-mato-grossense 2013/2014. Campo Grande, MS: SENAR/MS; FAMASUL, 2015. 494 p.

INSTITUTO DE PESQUISA ECONÔMICAAPLICADA(IPEA), Desafio do desenvolvimento. Revista de informações e debates do Instituo de Pesquisa Econômica Aplicada. Ano 10, ed.78, 16/01/2014. Disponivel em: http://www.ipea.gov.br/desafios/index.php?option=com_content\&view $=\mathrm{a}$

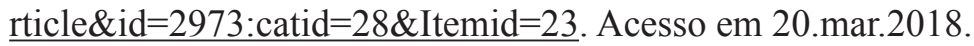

INTERNATIONAL ENERGY AGENCY (IEA). Decouplingof global emissionsandeconomicgrowthconfirmed. 2016. Disponível em: https:// www.iea.org/newsroom $/$ news $/ 2016 / \mathrm{march} /$ decoupling-of-globalemissions-and-economic-growth-confirmed.html. Acesso em: 05.abr.2018.

KANG, S.; DOH, S.; LEE, D.; LEE, D.; JIN, V.L. \& KIMBALL, J. Topographic and climatic controls on soil respiration in six temperate mixed-hardwoodforestslopes, Korea. Global Change Biology, Chichester, v. 9, p.1.427-1.437, 2003.

KASSNAYER, K.; NETO, H. J. F. A entrada em vigor do Acordo de Paris: o que muda para o Brasil? Textos para Discussão. Núcleo de Estudos e Pesquisa da Consultoria Legislativa. Senado Federal. Vol.215, 2016. Disponível em: <https://www12.senado.leg.br/publicacoes/estudoslegislativos/tipos-de-estudos/textos-para-discussao/td215> . Acesso em 04.maio.2018.

KRAFT, J; KRAFT, A. On the Relationship between Energy and GNP. In: Journal of Energy and Development, Ed.3, pg. 401-403, 1978.

LA SCALA, N.; DE FIGUEIREDO E. B., PANOSSO A. R. A review on soil carbonic cumulation due to the management change of major Brazilian agricultural activities. Brazilian Journal of Biology, São Carlos, v. 72, n.3, p. $775-785,2012$.

LEE, C.C. Energy consumption and GDP in developing countries: 
A cointegrated panel analysis. In: Energy Economics, 2005. Disponível em: $<\underline{\text { https://econpapers.repec.org/article/eeeeneeco/ }}$ v 3a27 3ay 3a2005 3ai 3a3 3ap 3a415-427.htm>. Acesso em: 03.maio.2018.

LEE, C.C. The Causality Relationship between Energy Consumption and GDP in G-11 Countries Revisited. Energy Policy, 34, no. 4. UK: Elsevier, 2006. Disponível em: https://www.sciencedirect.com/science/article/pii/ S030142150500128X. Acesso em: 07.maio.2018.

MINISTÉRIO DA CIÊNCIA, TECNOLOGIA, INOVAÇÕES E COMUNICAÇÕES (MCTIC) E ONU MEIO AMBIENTE. Trajetórias de mitigação e instrumentos de políticas públicas para alcance das metas brasileiras no Acordo de Paris. Pag. 15, Brasilia/DF, 2017. Disponível em: http://sirene.mcti.gov.br/documents/1686653/2098519/TrajetoriasEbook-b final.pdf/29c11698-b71d-4009-850c-a162090e1108. Acesso em 07.naio.2018.

MINISTÉRIO DO MEIO AMBIENTE (MMA), REPÚBLICA FEDERATIVA DO BRASIL PRETENDIDA, CONTRIBUIÇÃO NACIONALMENTE DETERMINADA PARA CONSECUÇÃO DO OBJETIVO DA CONVENÇÃO-QUADRO DAS NAÇÕES UNIDAS SOBRE MUDANÇA DO CLIMA, 2016. Disponível em: $<\underline{\text { http:// }}$ www.mma.gov.br/images/arquivo/80108/BRASIL $\% 20 \mathrm{iNDC} \% 20$ portugues\%20FINAL.pdf> . Acesso em: 04.mao.2018.

ORGANIZAÇÃO DAS NAÇÕES UNIDAS (ONU). Disponível em:

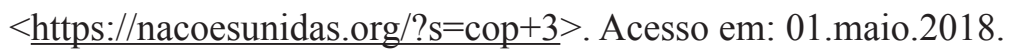

ORGANIZAÇÃO DAS NAÇÕES UNIDAS PARA EDUCAÇÃO, CIÊNCIA E A CULTURA (UNESCO). Biosphere Reserve Information The Pantanal Biosphere Reserve. MAB - Man and Biosphere Programme. Biosphere Reserves Directory, 2000. Disponível em: $<$ http://www.unesco. $\mathrm{org} / \mathrm{mabdb} / \mathrm{br} / \mathrm{brdir} /$ directory $/$ biores $. \mathrm{asp} ? \mathrm{code}=\mathrm{bra}+03 \& \operatorname{mode}=\mathrm{all}>$. Acesso em: 02.maio.2018.

Relatório anual GREENPEACE, 2009. Disponível em: <http://www. greenpeace.org/brasil/Global/brasil/report/anual/2009.pdf > Acesso em: 02.maio.2018. 
SARTORI, F.; LAL, R.; EBINGER, M. H.; PARRISH, D. J. Potential soil carbon sequestration and $\mathrm{CO}_{2}$ offset by dedicated energy crops in the USA. Critical Reviews in PlantSciences, New York, v. 25, n. 5, p. 441-472, 2006.

SGS - Sistema Gerador de Séries Históricas. Banco Central do Brasil. Disponível em <https://www3.bcb.gov.br/sgspub/localizarseries/ localizarSeries.do?method=prepararTelaLocalizarSeries $>$. Acesso em: 14.mai.2018.

SHAARI, M.S.; RAZAK, N.A.A.; BASRI, B.H.; The effects of electricity consumption and economic growth on Carbon Dioxide emission. In: International Journal of Energy Economics and Policy, vol. 7, 2017. Disponível em: < http://econjournals.com/index.php/ijeep/article/ view/5279>. Acesso em: 03.maio.2018.

SIGA-MS. Sistema de Informação Geográfica do Agronegócio de Mato Grosso do Sul. 2016. Disponível em: <http://www.sigaweb.org/ms/ sistema>. Acesso em: 01.abr.2018.

Sistema de Estimativas de Emissões e Remoções de Gases de Efeito Estufa (SEEG). Disponível em: http://plataforma.seeg.eco.br/total emission. Acesso em: 01.maio.2018.

TIWARI, A.K., Energy consumption, $\mathrm{CO}_{2}$ emissions and economic growth: A revist of the evidence from India. In: Applied Econometrics and International Development. Vol.11-2, 2011. Diposnivel em: http://www. usc.es/economet/journals1/aeid/aeid11212.pdf. Acesso em: 07.mar.2018.

ZHANG, X.P.; CHENG,X.M.; Energy consumption, carbono emissions, and economic growth in China. In: Econ Papers, Economics at your fingertips. Vol.68, 2009. Disponível em: https://econpapers.repec.org/ article/eeeecolec/v_3a68_3ay_3a2009_3ai_3a10_3ap_3a2706-2712.htm. Acesso em: 02.maio.2018.

\section{Como citar este artigo (ABNT):}

KUNIMOTO, Simone Yukimi; BOSON, Daniel Silva; OLIVEIRA, Michel Angelo Constantino de; MENDES, Dany Rafael Fonseca. Impacto do Desenvolvimento Econômico nas Emissões de CO2: Uma Aplicação da Curva Ambiental de Kuznets para o Mato Grosso do Sul. Veredas do Direito, Belo Horizonte, v. 15, n. 33, p. 321-345, set./dez. 2018. Disponível em: $\quad<$ http://www.domhelder.edu.br/revista/index.php/veredas/article/ view/1322>. Acesso em: dia mês. ano.

Artigo recebido em: 28/07/2018

Artigo aceito em: 20/11/2018 